\title{
Un Acercamiento a la Formación de Docentes como Investigadores Educativos en México
}

\author{
An Approach to the Training of Teachers as Educational \\ Researchers in Mexico
}

\author{
Evangelina Cervantes * \\ Universidad Autónoma de Ciudad Juárez, México
}

\begin{abstract}
En el presente artículo se analiza la formación de investigadores educativos tomando como referencia los procesos de formación docente en que participa el profesorado mexicano. Con base en la revisión de la política pública de formación y desarrollo profesional docente, así como diversas aportaciones teóricas sobre la temática, se distinguen las principales acciones gubernamentales que se han implementado para promover entre los docentes las habilidades para usar, aplicar y realizar investigación educativa. Mediante una metodología cualitativa, de corte documental, el análisis versa sobre el alcance conceptual de la formación de investigadores educativos, el modo en que ésta se ha realizado y, las acciones implementadas en la formación docente para incorporar la formación de investigadores. Se concluye el papel clave de la investigación en la transformación de la práctica docente, además se identifican en la política educativa diversos momentos y estrategias para formar a los docentes como investigadores, en donde el posgrado tiene una posición estratégica. Si bien, se observan ciertos avances, las oportunidades que se ofrecen al profesorado de educación básica para formarse como investigadores educativos son escasas, por lo que se requiere avanzar en la conformación de una masa crítica de docentes con las competencias necesarias para investigar. Se propone impulsar la investigación participativa que conduzca a acciones transformadoras y otorguen al profesorado una participación auténtica.
\end{abstract}

Descriptores: Educación básica; Formación de docentes; Formación de investigadores; Investigación pedagógica; Práctica pedagógica.

In this article, the training of educational researchers is analyzed taking as reference the processes of teacher training in which Mexican teachers participate. Based on the review of the public policy of teacher training and professional development, as well as various theoretical contributions on the subject, the main government actions that have been implemented to promote among teachers the skills to use, apply and carry out educational research are distinguished. By means of a qualitative methodology, of documentary nature, the analysis deals with the conceptual scope of the training of educational researchers, the way in which this has been carried out, and the actions implemented in teacher training to incorporate the training of researchers. It concluded the key role of research in the transformation of teaching practice, also identified in the educational policy various moments and strategies to train teachers as researchers, where the postgraduate has a strategic position. Although some progress is being made, the opportunities offered to basic education teachers to train as educational researchers are scarce, so it is necessary to advance in the formation of a critical mass of teachers with the necessary skills to investigate. It is proposed to promote participatory research that leads to transformative actions and gives to teachers an authentic participation.

Keywords: Basic education; Teacher education; Research training; Educational research; Teaching practice.

*Contacto: evangelina.cervantes@uacj.mx

ISSN: 1696-4713

www.rinace.net/reice/

revistas.uam.es/reice
Recibido: $\quad 25$ de marzo 2019

$1^{\text {a }}$ Evaluación: 4 de junio 2019

$2^{\text {a }}$ Evaluación: 27 de junio 2019

Aceptado: $\quad 10$ de julio 2019 


\section{Introducción}

En el presente artículo se analiza la formación de investigadores educativos tomando como referencia los procesos de formación docente en que participa el profesorado mexicano. Con base en la revisión de la política pública de formación y desarrollo profesional docente, así como diversas aportaciones teóricas sobre la temática, se distinguen las principales acciones gubernamentales que se han implementado para promover entre los docentes las habilidades necesarias para usar, aplicar y realizar investigación educativa. En este sentido, el análisis versa sobre tres preguntas: la primera refiere a la necesidad de aclarar el alcance conceptual de la formación de investigadores educativos; la segunda es una invitación para describir el modo en que ésta se ha realizado en el contexto mexicano; y, la tercera busca analizar las acciones implementadas en la formación docente-inicial y continua- para incorporar la formación de investigadores. El trabajo contribuye a la discusión sobre la formación de docentes como investigadores educativos a fin de identificar nuevos horizontes sobre el tema.

Si bien, la investigación en el aula -también llamada investigación de la práctica, investigación docente, investigación-acción participativa en educación, entre otros-, ha sido estudiada ampliamente por las posibilidades que ofrece para analizar, indagar y reflexionar sobre los procesos que se desarrollan en ella, en el presente trabajo la reflexión gira alrededor de su papel en la formación de investigadores educativos como una cuestión vinculada a los procesos de formación docente en México. Históricamente, la investigación en y de los espacios escolares se ha constituido en una estrategia recurrente en la formación del profesorado con la intención de "pasar del modelo de la racionalidad técnica a prácticas reflexivas alternas” (Hinojosa y Rodríguez, 2017, p. 116), en apego a los postulados del modelo crítico o alternativo de formación docente (Porlán, 1987).

En el país, los primeros acercamientos a la formación de investigadores educativos se ubican en la década de los sesenta. Desde entonces ha "transitado de una formación... que se podría considerar artesanal e incluso accidental, a un proceso de mayor sistematicidad" (Jiménez y Díaz-Barriga, 2012, p. 37), usualmente vinculada a la figura del profesorado de educación básica, y este a las condiciones políticas del momento. Así, a partir de los años setenta, la Secretaría de Educación Pública (SEP) ha incorporado la investigación en el aula en "todos los programas de formación docente como la manera "oficial" de hacer investigación” (Flores-Kastanis, Montoya-Vargas y Suárez, 2009, p. 293).

La propuesta gubernamental parte del supuesto de que, cuando los docentes se forman como investigadores educativos inician un proceso de autodescubrimiento que los lleva a la reconfiguración de creencias y a la transformación de su práctica docente (SEP, 2012). Con ello se espera resolver una serie de desafíos, entre ellos: la brecha entre la práctica docente y la investigación educativa; el empirismo docente; la ausencia de la cultura del uso de los resultados de la investigación, y la inercia en las estrategias de formación continua, entre otros.

Desde la posición de Murillo y Martínez-Garrido (2019), para eliminar la brecha entre la práctica docente y la investigación educativa, en América Latina se requiere analizar la correspondencia entre la investigación y las necesidades sentidas por docentes, directivos y tomadores de decisiones en política educativa. Al respecto, Zorrilla (2010) señala que, en México, la investigación educativa y la práctica docente 
viven en mundos separados, incluso podemos decir que en mundos paralelos. La investigación, a decir de los docentes, vive en su preciosa torre de marfil sin que se digne a mirar lo que acontece en las aulas y en las escuelas. (Zorrilla, 2010, p. 81)

Sobre este punto, el gobierno mexicano reconoce como un reto importante atender "el eventual desconocimiento y la falta de fundamentación en los resultados de la investigación científica en las acciones de planificación, toma de decisiones e intervención educativa en las instituciones de educación básica” (SEP, 2012, p. 3).

Además, la práctica docente comúnmente se justifica en el empirismo docente, es decir, "se realiza sin fundamento teórico, sin referencia científica, convirtiéndose en una práctica no validada, que se desvanece al término de cada ciclo escolar... [y] se sostiene de la percepción y del recuerdo personal, de las creencias vigentes en el docente" (Gavotto, 2014, p. 12). Esta ausencia de fundamento teórico se traduce como una debilidad en el camino hacia la mejora de la práctica docente que puede ser atendida al convertir a los docentes en usuarios de la investigación. En la retórica gubernamental se espera que los docentes sean tanto productores de conocimiento como usuarios estratégicos de los resultados de la investigación, lo que implica transitar de un modelo donde los resultados de la investigación son un referente exclusivo para los investigadores y tomadores de decisiones a un modelo donde esta información se coloca al alcance de los docentes para su revisión y análisis (Cervantes y Gutiérrez, 2018); se trata de una investigación situada que emerge y cobra sentido en el aula y la escuela. Para la SEP (2017) la investigación educativa constituye un insumo que permite "trazar pautas que orienten a las comunidades educativas en la planeación e implementación del currículo” (p. 30).

En la trasformación de la práctica docente se recurre a la formación continua, en donde la investigación en el aula se propone como una estrategia para dinamizar la forma en que los docentes desarrollan o renuevan sus habilidades, intercambian experiencias, confrontan sus propios saberes, nutren su práctica y se reconocen como agentes de cambio. Para Mendoza y Roux (2015) se trata de una "modalidad de desarrollo profesional innovadora... que apuesta a la participación activa de los maestros en procesos de investigación en el aula encaminados a resolver problemáticas específicas y contextualizadas" (p. 70). Con ella, se espera erradicar la inercia que provoca la forma en que, hasta hoy, se realiza la formación permanente del profesorado.

En general, la investigación en el aula refiere a una modalidad de investigación en la que "el profesor ha de reflexionar sistemáticamente sobre su práctica, sobre su trabajo en el aula y en su centro, y a partir de esta metodología reflexiva y crítica, participar en la transformación de la realidad educativa que le rodea" (Tójar, 2006, p. 112). Así, surge la figura de docente-investigador para aludir a un nuevo conjunto de competencias a cargo de los docentes, que incluye observar su práctica, reflexionar sobre ella, identificar áreas de desarrollo y diseñar una ruta de mejora. Este perfil, centrado en la investigación, reconoce en el docente "su capacidad transformadora... que pone más el énfasis en el proceso, en el contexto, en el diseño de su trabajo" (p. 112), así como la importancia de sus saberes.

Ante la propuesta de promover la figura del docente-investigador, se identifican dos posiciones: a favor se argumenta sobre la necesaria actualización de los saberes del profesorado, así como sus beneficios, en tanto que "cuando los maestros investigan los fenómenos que acontecen en sus propias aulas, se vuelven más críticos y reflexivos, generan conocimiento práctico y útil que potencializa la mejora de su práctica docente y 
se renuevan continuamente como profesores" (Mendoza y Roux, 2015, p. 49); y, en contra, se enfatizan las diferencias sustanciales entre la enseñanza y la investigación, como "dos actividades muy diferentes que demandan formación, tiempos, capacidades y motivaciones distintas" (Zorrilla, 2010, p. 82).

En el marco de la política educativa, para guiar al profesorado a la investigación en el aula y, con ella, promover la trasformación de la práctica pedagógica se proponen dos estrategias: la habilitación de los docentes como investigadores que les permita "diseñar, conducir y analizar diversas indagaciones en el propio contexto donde enseñan" (SEP, 2012, p. 4); y, "Alinear tanto la formación continua de maestros como la formación inicial" (SEP, 2017, p. 40). Las acciones que se han implementado para su cumplimiento constituyen el centro del ejercicio analítico que se presenta.

\section{Revisión de la literatura}

Una primera aproximación al estudio de la formación de investigadores educativos en México es la realizada, a finales de los ochenta, por Arredondo y colaboradores (1989) en la que distinguían entre procesos formales, asociados al posgrado donde se pretendía esa formación a través de propuestas curriculares intencionadas y estructuradas académicamente; $\mathrm{y}$, procesos no formales o informales, que se suscitaban de forma relativamente espontánea y en torno a la práctica. Por su parte, Martínez-Rizo (1997) señala que, en este periodo, frente a la expresión docente-investigador surgieron dos tendencias: una, en sentido amplio, que incluía tanto a investigadores universitarios como a docentes interesados en superarse e innovar sus prácticas; y otra, en sentido estricto, que agrupaba a aquellas personas con "una producción escrita significativa en publicaciones de nivel internacional” (p. 29). Al respecto, el autor propone una tercera tendencia de carácter conciliadora desde la cual solo será investigador educativo "quien se dedique profesionalmente de manera principal y habitual a la realización de estudios sistemáticos sobre temas educativos" (p. 71).

Moreno-Bayardo (2003) distingue entre formación para la investigación y formación para la investigación educativa. Con la primera expresión alude, en general, a un proceso que,

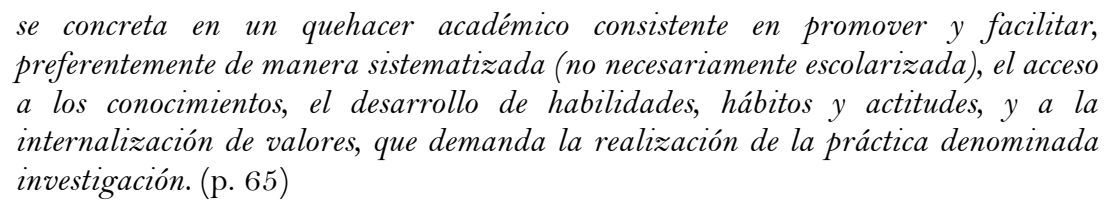

Mientras, con la segunda se refiere a la formación que, en particular, tiene como propósito:

a) Formar usuarios de investigación, dirigida a desarrollar en la persona los elementos necesarios para comprender los resultados de la investigación, ya sea para analizar sus hallazgos o valorar la posibilidad de transferencia; b) Formar personas para aplicar resultados de investigación con la intención de solucionar problemas, innovar prácticas o generar proyectos educativos; o, c) Formar investigadores educativos que se distinguen por "conocer profundamente el campo de la educación, tienen acceso a formación especializada... comúnmente en posgrados en educación” (p. 68).

En el contexto mexicano, el origen de la formación de investigadores educativos "no ocurrió como un proceso formalmente diseñado desde el currículum; por el contrario, se trata de un proceso tardío, como la misma conformación del campo de la disciplina" (Jiménez y Díaz-Barriga, 2012, p. 15). Así, las primeras huellas de este tipo de formación 
se ubican en el marco de los estudios etnográficos realizados en la década de los ochenta para observar y comprender los procesos de la vida escolar (Rockwell, 1995). En esta mirada a la escuela, se encontró en la etnografía "la mejor manera de documentar el modo de vida en este lugar tan cercano y a la vez tan ignorado" (p. 8), además ofreció la oportunidad de visibilizar a los sujetos que participaban en la construcción de la vida escolar; de analizar lo que ocurría al interior del aula; de abrir un espacio para que los alumnos expresaran e interpretaran el conocimiento escolar, en tanto los maestros transformaban su práctica cotidiana. La conformación de equipos de investigación, que incluían a investigadores y estudiantes del Departamento de Investigaciones Educativas del Instituto Politécnico Nacional, alude a las primeras experiencias de formación de investigadores educativos que se gestaron al interior del proyecto "La escuela cotidiana", coordinado por Elsie Rockwell y Justa Ezpeleta (Rockwell, 1995). En la revisión que Murillo y Martínez-Garrido (2019) hacen sobre la investigación educativa en América Latina, este hecho coincide con la etapa "de mayor auge y reconocimiento social... caracterizada por dar especial atención al contexto, tanto a nivel macroestructural, como a las dinámicas internas de las aulas" (p. 6).

Con una mirada retrospectiva, Schmelkes y colaboradores (2017) analizan la investigación que sobre la formación de investigadores educativos se generó en México durante el periodo del 2010 al 2016 a fin de identificar sus principales retos. Del ejercicio concluyen que, aun cuando la formación de investigadores en el país es un tema que ha cobrado relevancia desde hace más de cuatro décadas y ha sido abordada desde diversas perspectivas, presenta múltiples desafíos, como: la claridad conceptual de la noción de investigador educativo, la calidad de los procesos de formación en investigación, los aspectos éticos en la formación de investigadores, el papel de la tutoría, la identidad de los investigadores educativos, la evaluación de los procesos investigativos, el desarrollo de competencias para la investigación, las políticas de financiamiento, la conformación de redes, la atención de necesidades sociales a través de la investigación, entre otros. Para las autoras, delimitar el significado de investigador educativo constituye el punto de partida de cualquier ejercicio sobre el tema, ya que

a partir de esa conceptualización es que se orientan los esfuerzos de formación, se
definen los procesos desde donde se enseña a hacer investigación, se identifican y
seleccionan a los responsables de los procesos de formación quienes deben responder a
una serie de requisitos acordes a la definición misma de investigador. (p. 5)

Mientras en la región, la formación de investigadores se vincula frecuentemente con la educación superior, con una actividad exclusiva de aquellos con grado de doctor y que solo es realizable en el posgrado; en atención a los fines del presente trabajo se asume la propuesta de Moreno-Bayardo (2003) para quien ésta "necesita ser un objetivo que esté presente y sea atendido en todos los niveles” (p. 63), en especial, en la formación del profesorado. El ejercicio se inspira en la idea del profesor como investigador, propuesta por Stenhouse y Elliot, que incorpora nuevas competencias al perfil del docente, quien "no solo se dedica a la enseñanza sino que, como parte sustantiva de la misma, ha de observar y reflexionar sobre sus tareas, poniendo en cuestión todo aquello que realiza, como un paso previo a la mejora educativa" (Tójar, 2006, p. 112). Desde una concepción amplia de la formación de investigadores educativos, se promueve un "modelo de profesor que investiga, sólo o con la ayuda de observadores expertos, para resolver problemas concretos... y, paralelamente reflexionar, teorizar y reconstruir progresivamente el currículum" (Porlán, 1987, p. 27). 


\section{Método}

El trabajo se realizó en apego a la investigación cualitativa de corte documental, entendida como aquella en donde "el tema y la perspectiva de trabajo la construye el investigador de acuerdo a la naturaleza de las preguntas que se ha formulado y a la viabilidad y accesibilidad de la documentación disponible" (Galeano, 2004, p. 31). El procedimiento analítico se desarrolló en dos momentos, según la propuesta de Londoño, Maldonado y Calderón (2014). La etapa heurística consistió en la búsqueda de material primario en torno a los términos de 'formación docente' y 'formación de investigadores educativos' en México, principalmente en la Red de Revistas Científicas de América Latina y el Caribe, España y Portugal (Redalyc) y en el buscador de Google Académico, localizando siete artículos de investigación, una ponencia, cinco libros y cinco documentos publicados por la SEP. El ejercicio implicó la lectura inicial de dichos documentos a fin de extraer los datos principales y someterlos a un proceso de revisión que se organizó en torno a las nociones conceptuales sobre la formación de investigadores educativos, antecedentes en el contexto mexicano, vinculación con la formación inicial y continua.

La etapa hermenéutica, que refiere a la "capacidad para explicar, traducir, interpretar y explicar las relaciones existentes entre un hecho y el contexto en el que acontece” (p. 23), implicó la lectura, análisis y clasificación de la información, para su posterior interpretación crítica. En conjunto, la búsqueda de documentos oficiales sobre la formación docente vinculada a la investigación; la revisión exhaustiva de los textos normativos y discursos de las autoridades educativas en torno a las categorías de análisis y la extracción de información permitió responder al qué, cómo y por qué de la formación de docentes como investigadores educativos, así como construir un referente teórico para examinar las principales acciones gubernamentales implementadas en este rubro.

\section{Resultados}

En México, la formación docente constituye una responsabilidad del Estado, quien tiene la obligación legal de establecer líneas de formación en correspondencia a los "temas prioritarios de la política educativa actual... y otros temas de relevancia social, considerados como ejes transversales que contribuyen a la mejora de la calidad de la educación básica” (SEP, 2018, p. 7). En esta direccion, la Ley General de Educación de 2018 impone a las autoridades educativas la constitución del Sistema Nacional de Formación, Actualización, Capacitación y Superación profesional docente que se encargará, entre otras acciones, del desarrollo de la investigación pedagógica y la difusión de la cultura educativa (Art. 20); por su parte, la Ley General del Servicio Profesional Docente (LGSPD) de 2013 señala la responsabilidad del Estado de proveer cursos, investigaciones aplicadas y estudios de posgrado al personal docente (Art. 59); con la intención de contribuir en la mejora de la calidad educativa, responder a las necesidades de desarrollo del profesorado y atender las necesidades de la escuela y de la zona escolar (Art. 60). En general, la formación de docentes en torno a la investigación educativa se observa en tres momentos: la formación inicial, la formación continua y la superación profesional, como extensión de la segunda. Cada momento constituye un escenario particular que refleja el qué, cómo y para qué de esta formación. 


\subsection{La formación de investigadores educativos en la formación inicial}

Históricamente, la formación inicial de docentes en México se ha caracterizado por incorporar espacios curriculares para promover la observación y el análisis de la práctica docente, sin embargo, desde la reforma curricular de la educación normal, iniciada en los ochentas y formalizada en el 2012, estos espacios se establecen con la intención de reflexionar, investigar e innovar la docencia. En el caso de las Escuela Normales, en el 2012 se incorpora a los planes de estudio de la Licenciatura en Educación Preescolar y la Licenciatura en Educación Primaria el curso "Herramientas básicas para la investigación educativa" que, tiene como propósito "fomentar en el docente en formación un razonamiento reflexivo, sistemático, planificado y riguroso que le permita sustentar su práctica educativa, basada tanto en los avances de la investigación como en el uso de metodologías e instrumentos" (SEP, 2012, p. 2). El curso se centra en la revisión de las características del enfoque cuantitativo y cualitativo de la investigación, con el objetivo de que cada estudiante defina un problema, elabore instrumentos y analice la información recabada, a fin de desarrollar "la capacidad de valorar críticamente la pertinencia de la investigación educativa en pro de fundamentar su quehacer y toma de decisiones utilizando marcos teóricos y herramientas básicas de indagación sustentadas científicamente" (p. 4).

En el caso concreto de la formación inicial, ya sea aquella ofertada por las Escuela Normales o por las Universidades, se ofrece al docente en formación una aproximación a la compleja tarea de investigar en educación, usualmente mediante la realización de algunos cursos tendientes al diseño, implementación y elaboración de un informe de investigación o intervención. Desde la posición de Moreno-Bayardo (2003) podría tratarse de un tipo de formación para la investigación, de mediano alcance, dirigida a "quien necesita dicha formación como apoyo para un mejor desempeño de su práctica profesional o a quien solo necesita ser un buen usuario de investigación" (p. 64).

Desde la formación inicial se observa un primer desafío, mientras en el currículo se insiste en el desarrollo de competencias orientadas al uso permanente de la investigación educativa para enriquecer la práctica docente, una vez concluida la licenciatura, los docentes "olvidan toda recomendación de analizar y sistematizar sus prácticas... [es hasta que] realizan algún posgrado o especialización educativa, cuando nuevamente hacen observación de su práctica o realizan investigación de la misma” (Hinojosa y Rodríguez, 2017, p. 8).

\subsection{La formación de investigadores educativos en la formación continua}

En el contexto nacional, la formación continua, permanente o en servicio alude al "conjunto de actividades que permiten desarrollar nuevos conocimientos y capacidades a lo largo del ejercicio profesional y perfeccionarse después de la formación inicial" (SEP, 2018, p. 102), que incluye la capacitación, actualización y el desarrollo profesional. En general, ésta se realiza sobre la idea de formar-se desde lo individual y lo colectivo, por lo que se plantea en un "modelo dual de formación continua para aproximarse a la formación desde dos campos, definidos como 'fuera' y 'dentro' de la escuela” (Cordero et al., 2017, p. 25). En la Estrategia Nacional de Formación Continua, con este tipo de formación se busca favorecer en el profesorado sus capacidades, su desempeño en el aula y en la escuela; además, "tiene como propósito brindar herramientas de apoyo a quienes participarán en la Evaluación del Desempeño como parte del mecanismo de Permanencia y Promoción” (p. 17). 
En la lógica de la formación fuera de la escuela se recurre a un modelo centrado en el diseño e implementación de cursos de actualización que se ofrecen en dos modalidades: a) Cursos en cascada, en donde la SEP "instruye a un grupo de profesores sobre las innovaciones educativas y, posteriormente, sus integrantes se convierten en capacitadores e imparten los cursos a otros colegas" (Mendoza y Roux, 2015, p. 44); y, b) Cursos a la carta, a partir del 2006, con la Reforma Integral de la Educación Básica (RIEB) se incluyó a las universidades y centros de investigación entre las Instituciones Especializadas en Formación Pedagógica de Profesionales de la Educación (IEFP), encargadas del diseño y operación de la oferta de formación continua, actualización y desarrollo profesional docente.

En esta estrategia cada IEFP, a través de un equipo de especialistas, diseña un conjunto de cursos, preferentemente en línea y en apego al contenido de la reforma, para atender la demanda del profesorado. Sobre el tema, Cuevas (2019) advierte sobre el pernicioso hecho de conformar todo un conjunto de dispositivos - guías, simuladores de exámenes y cursospara vincular la formación a la evaluación, y observa, en lo anterior, la tensión entre "formar a un profesional vs. formar a un trabajador para que apruebe un examen" (p. 220). De este modo, la política de formación continua ha quedado en deuda, ya que "la influencia de los cursos en la mejora de la práctica docente es poca o inexistente, porque se imparten en espacios descontextualizados, alejados de las vicisitudes de la enseñanza en el aula" (Mendoza y Roux, 2015, p. 46).

La Estrategia Nacional (2018), con efectos en los ciclos escolares 2017-2018 y 2018-2019, se desarrolló en tres líneas:

- Formación para el diseño de proyectos correspondientes a cada figura educativa, en esta línea se pretende que el personal educativo cuente con "conocimientos, capacidades y aptitudes para la mejora continua de su práctica educativa y el máximo logro de aprendizajes de los estudiantes... [y] tenga mayores posibilidades de consolidar su compromiso profesional y su quehacer educativo en la escuela" (p. 17).

- Atención al personal educativo que participa en los mecanismos de evaluación, esta línea alude a la atención de las necesidades del personal docente "para el fortalecimiento de las prácticas de enseñanza, el desarrollo profesional y su impacto en la mejora del aprendizaje de los alumnos para el logro de la calidad educativa" (p. 18).

- Formación continua en temas prioritarios de la reforma, así como en temas transversales socialmente relevantes, esta línea se orienta al fortalecimiento de "los ámbitos educativos de conocimientos pedagógicos, disciplinarios, de gestión escolar y temas transversales que contribuyan al desarrollo personal y social que requiere el personal educativo para la mejora de su práctica" (p. 23).

Una característica de la Estrategia Nacional, según sus autores, es "ofrecer una oferta académica de formación continua pertinente y de calidad, centrada en los aprendizajes clave, en ambientes incluyentes, en el contexto específico de sus alumnos" (p. 22). Pese al mandato legal que insiste en el desarrollo de la investigación pedagógica, la única referencia a esta se encuentra en los proyectos de investigación que, como trayectos formativos, solo se ofrecen al personal de educación media superior y superior. 
La formación en la escuela refiere a una modalidad de formación colectiva en donde los propios docentes seleccionan tanto los contenidos como su desarrollo. Para Cordero y otros (2017), esta es "la aportación más innovadora de la LGSPD en materia de formación... en tanto que su objeto de mejora es la escuela en su conjunto" (p. 23). Esta formación in situ puede detonar el desarrollo de acciones afirmativas para el logro de la mejora escolar al promover entre el profesorado el intercambio de ideas, la elaboración colegiada de proyectos, el análisis de problemas comunes y la búsqueda de soluciones; además, dado su interés en el quehacer del aula puede promover procesos de investigación sobre la actuación del profesorado. En el marco de la Reforma Educativa, el Consejo Técnico Escolar (CTE) se concibe como el espacio colectivo de aprendizaje y desarrollo profesional, dirigido al "análisis y la toma de decisiones, a partir del desarrollo de estrategias y actividades didácticas que permitan la mejora de la práctica docente y de los aprendizajes de los estudiantes" (SEP, 2018, p. 48), por lo que puede ser el espacio que ofrece mayores oportunidades para promover la formación de los docentes como investigadores. Del mismo modo, la SEP (2017) propone la creación de redes de colaboración entre pares y con otros miembros de la comunidad, acción que puede emplearse para establecer alianzas con investigadores, quienes pueden "sumarse a la transformación de las escuelas. Sus iniciativas, publicaciones y demás acciones también abonarán a la reflexión acerca de cómo apoyar a la escuela a crecer y fortalecerse" (p. 39).

En resumen, se identifica un segundo desafío desde el que se cuestiona la pertinencia de la formación continua frente a la investigación. En la experiencia mexicana se observa una amplia oferta de cursos, talleres, diplomados, asesoría en el aula y en la escuela, con los que se busca responder a las necesidades de formación de los docentes en servicio que deja al margen la investigación como contenido curricular y como método de trabajo.

\subsection{La formación de investigadores educativos en la superación profesional}

En el contexto del profesorado de educación básica mexicano, el arribo al posgrado se realiza como la etapa más alta del desarrollo profesional docente, que comienza en la formación inicial, se fortalece en la formación continua -mediante la actualización y la capacitación- y se consolida en la superación profesional, entendida como aquella "formación destinada a quienes desean especializarse en campos diversos relativos al quehacer educativo y alcanzar mayores niveles de habilitación profesional y desarrollo... a través de estudios de especialización, maestría y doctorado (SEP, 2008, como se citó en Cordero et al., 2017, p. 45). Una vez concluida la licenciatura, algunos docentes se encuentran ante la decisión de continuar su formación ingresando a alguno de los programas de especialización, maestría y doctorado que se ofertan en la región. Los motivos de ingreso al posgrado son diversos, y pueden ser explicados antes y después de la Reforma Educativa, por ejemplo; previo a su implementación, y en apego al Programa Nacional de Carrera Magisterial'1, que premiaba la participación de los docentes en procesos de formación continua con estímulos económicos, el ingreso al posgrado significaba mayores ingresos y posibilidades de promoción, lo que derivó en un credencialismo patológico; por su parte, una vez implementada la Reforma, la entrada al posgrado se vinculó al fortalecimiento de los conocimientos frente a los procesos de

${ }^{1}$ El Programa Nacional de Carrera Magisterial inició en enero de 2003 como un sistema de estímulos para el mejoramiento de las condiciones de vida, laborales y educativas del profesorado de Educación Básica, concluyó en mayo del 2015 , al ser sustituido por el Programa de Promoción en la Función por Incentivos en la Educación Básica. 
evaluación -para el ingreso, la permanencia, la promoción y los incentivos-, lo que generó una reducción del papel de la formación, ahora sujeta a los contenidos y orientaciones de los procesos de evaluación docente. Con todo, ante la diversidad de razones para participar en el posgrado persiste la brecha entre la formación y la transformación de la práctica docente; ahora se tiene un mayor número de profesores con grado de maestría y doctorado, mientras la práctica docente se mantiene inalterada.

El posgrado constituye el lugar donde se realiza, de manera sistemática y escolarizada, la formación para la investigación. Para Moreno-Bayardo (2003) este tipo de formación se dirige "a quien se dedicará a la investigación como profesión" (p. 64); por lo que se trata de un proceso formativo "de amplio espectro, mediante el cual se preparan los agentes que tendrán como desempeño profesional la generación de conocimiento en un campo determinado" (p. 64). En el contex to nacional, se cuenta con una diversidad de posgrados en educación: de sostenimiento público o privado; pertenecientes a las Instituciones Formadoras de Docentes (IFD) u otras instituciones; en contextos presenciales o en línea; en modalidades escolarizadas o semiescolarizadas, adscritos o no al Programa Nacional de Posgrados de Calidad (PNPC); para estudiantes de tiempo parcial o completo, entre otras. Ante esta amplia oferta de posgrado, es necesario abrir "el debate hacia la calidad de la formación que se imparte en estos programas... ya que en muchos de ellos la prioridad es el cumplimiento de indicadores y no una sólida formación académica" (Jiménez y Díaz-Barriga, 2012, p. 17).

En el caso de los docentes de educación básica que desean avanzar en su desarrollo profesional, tal vez la decisión de mayor dificultad refiere a elegir entre los posgrados que se ofertan en las IFD u otras instituciones. Los primeros se caracterizan por estar avalados por la SEP y el Sindicato Nacional de Trabajadores de la Educación, lo que implicaba, hasta antes de la Reforma, el derecho a dos tipos de apoyos (Gobierno del Estado de Chihuahua, 2015): beca comisión, consistente en "la autorización de movimiento lateral que se otorga para realizar estudios de especialización, maestría, doctorado o posdoctorado” (p. 1), hasta por uno o dos años según corresponda; o, beca económica, consistente en "la autorización de una aportación económica a quienes inician o están cursando estudios de posgrado” (p. 1), con un monto equivalente al $25 \%, 33 \%$ o $50 \%$, ya sea que se trate de estudios de maestría, doctorado o posdoctorado. Sin embargo, con la implementación de la Reforma Educativa, las licencias para la realización de estudios de posgrado se tornan inviables a la luz de la LGSPD y el posgrado como estrategia formativa se diluye en los documentos oficiales. Para Cervantes y Anguiano (2017) en la actualidad, las IFD enfrentan una serie de retos de diversa índole: a) política, requieren tanto de un marco que defina su personalidad jurídica como de acciones concretas para articular su oferta a la Estrategia Nacional de Formación Continua; b) institucional, demandan planes de trabajo para cubrir los requisitos que exige el PNPC, así como promover la vinculación con otras instituciones, centros y redes de investigación; c) curricular, precisan programas flexibles, dinámicos y pertinentes a las necesidades del profesorado; $y, d$ ) de generación de conocimiento, urge promover la investigación, la intervención y la evaluación educativas, además de mecanismos para la socialización de estas experiencias.

Por otra parte, la oferta educativa de las universidades, adscrita al PNPC, destaca por cumplir con los más altos estándares de calidad y pertinencia (CONACYT, 2015a), y se distingue por contar con un sistema de becas para estudiantes de dedicación exclusiva consistentes en un apoyo económico mensual aplicable a la duración oficial del programa correspondiente a 4.0, 4.5 o 6.0 Unidades de Medida y Actualización -en la práctica su 
monto es equiparable al pago mensual de una plaza inicial de profesor de educación preescolar o primaria- y servicio médico a través del Instituto de Seguridad y Servicios Sociales de los Trabajadores del Estado. Además, la evaluación de estos programas prioriza la productividad académica, la movilidad de docentes y estudiantes en instituciones nacionales o internacionales, la colaboración en proyectos de investigación con académicos de diversas instituciones, así como la dirección de tesis que garantice la eficiencia terminal. Para Jiménez y Díaz-Barriga (2012) lo anterior ha suscitado el desarrollo de acciones dirigidas al cumplimiento de indicadores en un plazo determinado, lo que ha obligado a las instituciones

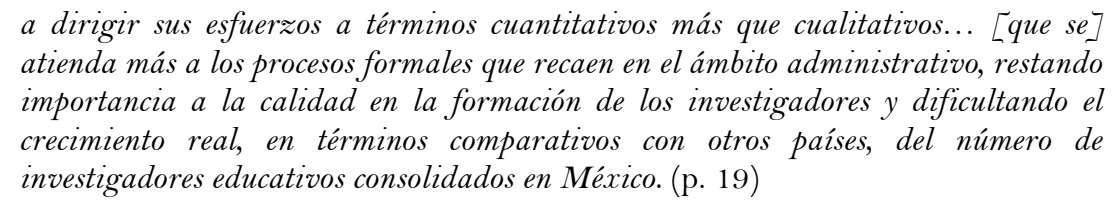

Mientras los programas de las IFD están orientados a la práctica profesional, la mayoría de los posgrados en educación del PNPC están orientados a la investigación a fin de "proporcionar al estudiante una formación amplia y sólida en un campo de conocimiento con una alta capacidad crítica y creativa a través de investigaciones originales” (p. 12). De la experiencia universitaria destaca la formación de investigadores educativos que se realiza mediante una asesoría personalizada, bajo la tutoría de un grupo de profesores con amplia trayectoria académica y experiencia en investigación, a partir de un conjunto de actividades como los coloquios semestrales, las estancias de movilidad académica y la escritura colegiada. Sin embargo, comúnmente los objetos de investigación surgen de la vida cotidiana, del diálogo con especialistas, de la lectura de los trabajos de diferentes investigadores, o de alguna experiencia significativa para el estudiante que con frecuencia se sitúa fuera de la escuela y el aula.

La lejanía entre la investigación y las necesidades del profesorado se presentan incluso en la investigación educativa financiada por el gobierno, por ejemplo, en la Convocatoria del Fondo Sectorial de Investigación para la Educación, el CONACYT (2015b) invitaban a los estudiosos de la realidad educativa a presentar propuestas de investigación que "permitan mejorar la política y programas de acción orientados al mejoramiento de la calidad, equidad y atención a la diversidad en los niveles, tipos y modalidades que constituyen la educación básica en México" (parr. 2); y que ofrezcan como resultado información pertinente a la "producción de materiales educativos, la actualización de profesores y directivos, los modelos educativos de atención a grupos sociales específicos, el mejoramiento de la gestión escolar y la efectividad social de la escuela mexicana, así como atender con pertinencia las condiciones y necesidades de educación de la población nacional" (párr. 3), sin explicitar el modo en que éstas prioridades se definieron y el tipo de participación del profesorado en ello. Al respecto, Murillo y Martínez-Garrido (2019) señalan que, es "fundamental que se refuerce el desarrollo de una investigación educativa propia, que recoja las problemáticas de sus escuelas, identifique sus propias estrategias para la mejora y promueva el desarrollo de políticas educativas contextualizadas a la realidad de sus aulas" (p. 24), lo que implica el diálogo con sus actores.

En la actualidad, los procesos de formación de investigadores educativos en el posgrado se pueden clasificar según se trate de las IFD y de aquellos adscritos al PNPC, ya sea por la dedicación parcial o exclusiva al programa, por la formación inicial de sus estudiantes, por los requisitos de ingreso, por los apoyos recibidos, por la cercanía con investigadores de 
mayor experiencia, por el contacto con la realidad escolar, por las expectativas laborales que se generan en ellos, entre otros. En el caso de las IFD, al posgrado ingresa un tipo específico de estudiante: docentes en servicio, con estudios en educación o profesionistas sin estudios pedagógicos que laboran en centros escolares públicos o privados en los diferentes niveles educativos, con disponibilidad de tiempo para atender tanto las exigencias de su plaza docente como los requerimientos del programa, sin apoyo económico -salvo aquellos que resultan beneficiados con alguna beca-, y con la expectativa común de enfrentar con mayores elementos los mecanismos de evaluación y promoción; mientras, en los posgrados adscritos al PNPC, el ingreso se caracteriza por ser estudiantes de tiempo completo, con formación en diversas disciplinas, frecuentemente sin experiencia docente, bilingües, hábiles en el manejo de las tecnologías y con un destino laboral vinculado a la investigación, ya sea como profesor-investigador en alguna institución de educación superior o como titular en algún centro de investigación.

\section{Discusión y conclusiones}

En el presente estudio se ofrece un panorama de la formación de investigadores educativos tomando como referencia los procesos de formación docente en que participa el profesorado mexicano. De inicio se concluye que formar a los docentes en investigación es necesario para animar el análisis, la reflexión y la transformación de su práctica; lo anterior implica transitar de una visión sobre la educación a una visión desde la educación; pasar de la idea del aula y la escuela como sistemas simples, a la idea donde se les reconoce como "una compleja malla de interacciones en diversos niveles (académicos, psicosocial, simbólico, etc.) y donde las variables contextuales dan sentido particular, en muchos casos, a los procesos que acontecen en el mismo" (Porlán, 1987, p. 31).

De entre los resultados encontrados se destacan algunas ideas relevantes: se requiere repensar la noción de formación de investigadores educativos, en principio considerar que no se puede hablar de investigación educativa al margen de la escuela y el aula, e invisibilizar el papel protagónico de los sujetos que les dan vida y sentido: los docentes. Una definición restrictiva de ella, como una tarea propia del posgrado, niega las posibilidades de formación en la práctica que ofrece, cotidianamente, el desempeño docente.

De la revisión de la política educativa se distinguen los diversos momentos y estrategias empleadas para promover en el profesorado las habilidades necesarias para usar, aplicar y realizar investigación educativa. Así, los docentes en formación tienen acceso a cursos con los que se espera desarrollar en ellos los conocimientos básicos sobre la investigación, a través de momentos curriculares para observar la práctica y diseñar proyectos. Sobre el punto, Jiménez y Díaz-Barriga (2012) advierten que "es necesario conocer métodos y técnicas, pero enfatizan que por sí mismos no orientan al estudiante a realizar una investigación, pues le proveen de un saber conceptual pero no de las habilidades inherentes al trabajo de investigar" (p. 34); por su parte, los docentes en servicio tienen a su alcance una amplia oferta educativa para formarse fuera o dentro de la escuela, ya sea al participar en espacios externos -cursos, talleres, especialidades, maestrías o doctorados-, o en espacios escolares -el CTE o las modalidades de acompañamiento docente-, la investigación, como contenido curricular o método de trabajo, es "una herramienta que contribuye a que los profesores identifiquen soluciones prácticas a las problemáticas 
concretas en el aula $[y]$ permite que los profesores monitoreen su desempeño desde una postura más autocrítica” (Mendoza y Roux, 2015, p. 57).

Respecto a las acciones implementadas en la formación docente destaca la superación profesional que se realiza en el posgrado, donde se observan dos vías: una, configurada en el marco de los programas de las IFD, donde los docentes participan con mayor frecuencia, pese a las debilidades institucionales, que se traducen en una débil formación para la investigación, y la falta de apoyos; y, otra, que tiene lugar en las instituciones con programas adscritos al PNPC, con procesos de calidad acreditada, diversos apoyos y un prometedor futuro como investigadores, pero alejados comúnmente del acontecer cotidiano en el aula. Ya sea por una u otra vía, la formación de docentes como investigadores educativos en el posgrado constituye un reto, sobre todo, ante la carencia en el país de una masa crítica de docentes que cuenten con las habilidades que la investigación educativa de calidad requiere. En este sentido, se necesitan programas de posgrado al alcance del profesorado de educación básica que contribuya al fortalecimiento de sus capacidades para realizar investigación.

Pese al reconocimiento oficial de la investigación educativa como "imprescindible para contribuir a una toma de decisiones informada en todos los niveles educativos: sistema educativo, escuela y aula" (Murillo y Martínez-Garrido, 2019, p. 23), éste no ha permeado la política pública de formación y desarrollo profesional docente. Aun cuando se observan ciertos avances, las oportunidades que se ofrecen al profesorado de educación básica para formarse como investigadores educativos son escasas. En esta dirección, Cuevas (2019) señala que el cambio en la formación docente es un proyecto lento

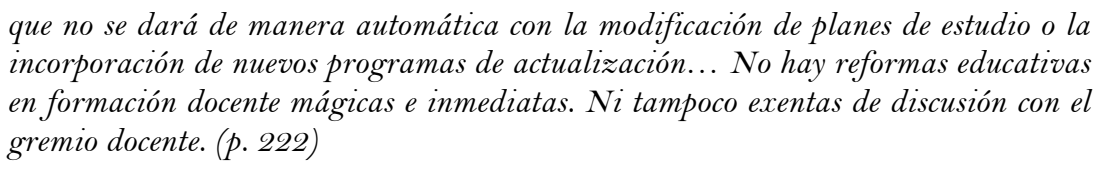

De este modo, cualquier propuesta requiere retomar la voz de los formadores docentes, de los gestores de políticas, de los expertos, de los maestros en servicio y de los estudiantes normalistas.

En el documento se rescató información desde la que se intentó responder al qué, cómo y por qué de la formación de investigadores educativos; a fin de contribuir a la discusión conviene centrar la reflexión final en el para qué, lo que ayudará a identificar nuevos horizontes sobre el tema. Del ejercicio se concluye la necesidad de impulsar la investigación realizada por los docentes para generar información relevante sobre la realidad educativa y, promover la formación de investigadores en apego a las "ideas sobre investigación participativa social y educativa entre académicos y personas 'comunes y corrientes', como una parte constitutiva de la práctica pedagógica de naturaleza emancipatoria” (Flores-Kastanis, Montoya-Vargas y Suárez, 2009, p. 295). Avanzar hacia procesos de formación desde los que se produzca conocimiento crítico, que conduzca a acciones transformadoras, requiere de experiencias formativas que otorguen al profesorado "una 'participación auténtica'... para producir conocimiento de manera colectiva sobre sus propios intereses, problemas y luchas” (p. 303). Se trata de una formación en investigación educativa comprometida con la justicia social.

Respecto a las limitaciones del estudio, el inconveniente principal alude a la escasa producción investigativa sobre el tema. Con frecuencia, la formación de investigadores educativos aparece como un tema periférico en los estudios sobre el posgrado en 
educación; y, su vinculación con la formación del profesorado de educación básica es incipiente, pese a los diversos esfuerzos para incluirla como contenido y método de trabajo. Por otra parte, la complejidad del sistema de formación docente en México, dado su contexto político, organizacional e institucional, hace difícil explicar el marco normativo y su concreción en las estrategias formativas en que participa el profesorado. Además, la experiencia de la autora en diferentes contextos de formación docente constituyó una tensión permanente para el análisis de las múltiples aristas del tema, la identificación de áreas críticas y la búsqueda de soluciones pertinentes. Finalmente, el estudio constituye un ejercicio diagnóstico sobre la formación de docentes en investigadores educativos que puede ser el punto de partida de futuras investigaciones sobre las condiciones en que se realiza la formación de investigadores educativos, las trayectorias académicas de docentesinvestigadores, los procesos de investigación suscitados en la escuela, las innovaciones en la formación de investigadores, los usos de la investigación educativa en la educación básica, entre otros dominios de problematización.

\section{Referencias}

Arredondo, V. M., Martínez, S., Mingo, A. y Wuest, T. (1989). Los procesos de formación y conformación de los agentes de la Investigacion Educativa. Cuadernos del CESU, 13, 1-66.

Cervantes, E. y Anguiano, B. (2017). Presente y futuro de los posgrados en Educación en el Estado de Chihuahua. En VVAA, Actas del $3^{\text {er }}$ Congreso internacional "Formación de profesionales de la educación: Perspectivas y desafíos emergentes" (pp. 1-9). Oaxaca: Universidad Autónoma Benito Juárez de Oaxaca.

Cervantes, E. y Gutiérrez, P. R. (2018). La supervisión escolar en la difusión y uso de la evaluación educativa en Chihuahua. Revista del Centro de Investigación y Docencia, 8, 7-21.

CONACYT. (2015a). Fondo sectorial de investigación para la educación. Convocatoria de investigación en educación básica. Recuperado de

https://www.conacyt.gob.mx/index.php/sni/convocatorias-conacyt/convocatoriasfondos-sectoriales-constituidos/convocatoria-sep-conacyt/convocatorias-seb-sepconacyt/convocatorias-cerradas-seb-sep-conacyt/convocatoria-2015-sep-seb

CONACYT. (2015b). Marco de referencia para la evaluación y seguimiento de programas de posgrados presenciales. Recuperado de https://www.conacyt.gob.mx/index.php/sni/convocatoriasconacyt/convocatorias-pnpc/marcos-de-referencia-pnpc

Cordero, G., Jiménez, J. A., Navarro, C. y Vázquez, M. (2017). Diagnóstico de la política pública de formación y desarrollo profesional del personal educativo de educación básica de la reforma educativa. Ciudad de México: Instituto Nacional para la Evaluación de la Educación.

Cuevas, Y. (2019). Los profesores del sistema educativo mexicano: Formación, actualización y prácticas académicas en educación básica y media superior. En A. Buendía y G. Álvarez, La investigación educativa ante el cambio de gobierno en México. Reflexiones y propuestas para el futuro (pp. 200-223). Ciudad de México: Consejo Mexicano de Investigación Educativa.

Flores-Kastanis, E., Montoya-Vargas, J. y Suárez, D. H. (2009). Investigación-acción participativa en la educación latinoamericana. Un mapa de otra parte del mundo. Revista Mexicana de Investigación Educativa, 14(4), 289-308.

Galeano, M. E. (2004). Diseño de proyectos en la investigación cualitativa. Medellín: Fondo Editorial Universidad EAFIT.

Gavotto, O. I. (2014). Transformando la educación, desde la práctica docente: Reflexionando en y sobre la acción. Bloomington, IN: Palibrio. 
Gobierno del Estado de Chihuahua. (2015). Convocatoria beca-comisión y beca económica 2015-2016. Chihuahua: Gobierno del Estado de Chihuahua.

Hinojosa, R. y Rodríguez, R. A. (2017). La educación preescolar en Chihuahua: Experiencias en la práctica y en la formación de sus educadoras. Chihuahua: ENSECH.

Jiménez, M. S. y Díaz-Barriga, Á. (2012). Posgrado en educación y la formación en investigación. El caso de la Universidad Autónoma de Tlaxcala. En A. Colina y Á. Díaz-Barriga, La formación de investigadores en educación y la producción del conocimiento (pp. 15-44). Ciudad de México: Ediciones Díaz de Santos.

Londoño, O. L., Maldonado, L. F. y Calderón, L. C. (2014). Guía para construir estados del arte. Bogotá: International Corporation of Network of Knowledge.

Martínez-Rizo, F. (1997). El oficio del investigador educativo. Aguascalientes: Universidad Autónoma de Aguascalientes.

Mendoza, J. L. y Roux, R. (2015). La investigación docente y el desarrollo profesional continuo: Un estudio de caso en el noreste mexicano. Innovación Educativa, 16(70), 43-60.

Moreno-Bayardo, M. G. (2003). Desde cuándo y desde dónde pensar la formación para la investigación. Nueva Época, 7(14), 63-81.

Murillo, F. J. y Martínez-Garrido, C. (2019). Una mirada a la investigación educativa en América Latina a partir de sus artículos. REICE. Revista Iberoamericana sobre Calidad, Eficacia y Cambio en Educación, 17(2), 5-25. https://doi.org/10.15366/reice2019.17.2.001

Porlán, R. (1987). Investigación en la escuela. Revista de Investigación e Innovación Escolar, 1, 1-22.

Rockwell, E. (1995). De huellas, bardas y veredas: Historia cotidiana en la escuela. En E. Rockwell (Ed.), La escuela cotidiana (pp. 13-57). Ciudad de México: Fondo de Cultura Económica.

Schmelkes, C., Mata, A., López, M. y Padilla, M. (2017). Retos y desafíos de los investigadores de la formación de investigadores en educación. En COMIE (Orgs.), Actas del XIV Congreso Nacional de Investigación Educativa (págs. 100-130). San Luis Potosí: Consejo Mexicano de Investigación Educativa.

SEP. (2012). Licenciatura en educación primaria. Plan de estudios 2012. Herramientas básicas para la investigación educativa. Ciudad de México: Secretaría de Educación Pública.

SEP. (2017). Aprendizajes clave para la educación integral. Plan y programas de estudio para la educación básica. Ciudad de México: Secretaría de Educación Pública.

SEP. (2018). Estrategia nacional de formación continua, 2018. Ciudad de México: Dirección General de Formación Continua, Actualización y Desarrollo Profesional de Maestros de Educación Básica.

Tójar, J. C. (2006). Investigación cualitativa: Comprender y actuar. Madrid: La Muralla.

Zorrilla, M. (2010). Investigación educativa, políticas públicas y práctica docente. Triángulo de geometría desconocida. REICE. Revista Iberoamericana sobre Calidad, Eficacia y Cambio en Educación, 8(2), 74-92.

\section{Breve CV de la autora}

\section{Evangelina Cervantes}

Profesora del Departamento de Humanidades de la Universidad Autónoma de Ciudad Juárez (UACJ) y Coordinadora de la Unidad Juárez del Centro Chihuahuense de Estudios de Posgrado. Doctora en Educación, Maestra en Desarrollo Educativo, Licenciada en 
Derecho y Licenciada en Educación Preescolar. Su trayectoria en investigación se centra en el trabajo y formación docente, política educativa y didácticas especializadas. Es autora de diversos libros, capítulos y artículos científicos relacionados con el área. Investigadora de diferentes proyectos de investigación a nivel local y estatal. Actualmente es miembro del Cuerpo Académico de Estudios de Educación y Ciencias Sociales. Coordinadora del Centro de Investigación Educativa de la UACJ. ORCID ID: http://orcid.org/0000-00016980-22 10. Email: evangelina.cervantes@uacj.mx 